phys. stat. sol. (a) $25, \mathrm{~K} 27$ (1974)

Subject classification: 10.1 and $14.1 ; 21$

Sektion Physik der Universität München

\title{
Galvanomagnetic Measurements of Annealing in Deformed Aluminium
}

By

C. RAU

In (1) we showed the usefulness of combined resistivity and magnetoresistivity measurements for the interpretation of the recovery of quenched-in defects in aluminium. At $T>400 \mathrm{~K}$ considerable annealing of the resistivity $\mathrm{g}_{\mathrm{o}}$ took place. The isochronal recovery was dominated by changes in the structure of the defects. The formation of larger defect aggregates was observed; they contributed only a small resistivity change.

In the following we present measurements on samples with defect aggregates introduced by deforming at concentrations higher than in the previous investigation. In particular, the high field dependence of the magnetoresistivity of defects in the region of self-diffusion is demonstrated.

We used $1 \mathrm{~mm}$ thick aluminium wires $(99.999 \%$, Degussa, Hanau) $10 \mathrm{~cm}$ in length. Cold working was performed during manufacture of the wires. They were annealed at room temperature. Potential leads were attached by spot welding. The resistivity ratio $R_{273 K} / R_{4 K}$ was approximately 270 .

The samples were isochronically step annealed with $2 \mathrm{deg} / \mathrm{min}$ and the galvanomagnetic resistivity $\varrho(\mathrm{H})$ and $\rho_{0}$ measured with an accuracy of $3 \times 10^{-13} \Omega \mathrm{cm}$.

After each annealing step the galvanomagnetic coefficient $P_{0}$ was determined:

$$
P_{0}\left(H / \rho_{0}\right)^{2}=\Delta \rho(H) / \rho_{0}=\rho(H) / \rho_{0}-1
$$

for $\mathrm{H} / \mathrm{\rho}_{\mathrm{O}}=0.8 \mathrm{kG} / \mathrm{n} \Omega \mathrm{cm}$ where $\omega \tau=0.2$, $\omega$ being the cyclotron frequency and $\tau \sim 1 / \rho_{0}$ the average time between two scattering events. In addition the normalized high field magnetoresistivity

$$
\mathrm{S}=\Delta \mathrm{\rho}(\mathrm{H}) / \mathrm{g}_{\mathrm{o}}
$$

was determined at $\mathrm{H} / \mathrm{g}_{\circ}=40 \mathrm{kG} / \mathrm{n} \Omega \mathrm{cm}$ where $\omega \tau \approx 10$.

The galvanomagnetic coefficient $P_{i}$ corresponding to impurity atoms was determined according to equation $(1)$ with the measured values $\mathrm{g}(\mathrm{H})$ and $\varrho_{\mathrm{O}}$ of samples which were 


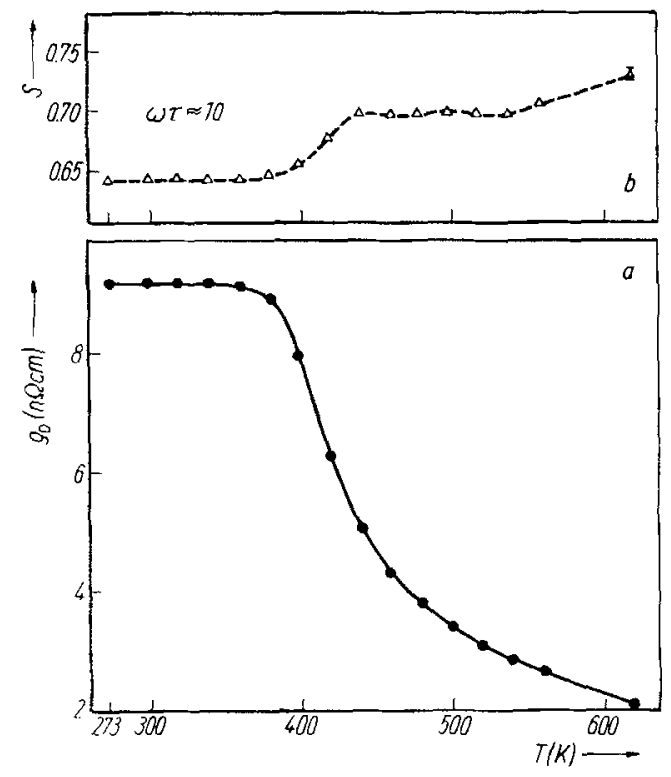

Fig. 1. a) Isochronal annealing curve of resistivity $\mathrm{g}_{\mathrm{O}}$ for a deformed $\mathrm{Al}$ wire of $1 \mathrm{~mm}$ diameter $(\Delta \mathrm{T}=20 \mathrm{~K}, \Delta \mathrm{t}=10$ min). b) Isochronal annealing of the high field galvanomagnetic coefficient $S$ at $\omega \approx 10$

fully annealed at $620 \mathrm{~K}$. It was assumed that the contribution to the magnetoresistivity from impurity atoms considerably exceeds the contribution from dislocations, grain boundaries, and sample size effect $(2,3)$. The resistivity $\rho_{0}$ at $4 \mathrm{~K}$ (after annealing at $620 \mathrm{~K}$ ) was approximately $1.9 \mathrm{n} \Omega \mathrm{cm}$. (The approximate relations are $1 \mathrm{ppm}$ impurity atoms $=1 \mathrm{n} \Omega \mathrm{cm}$ and $10^{9}$ dislocation lines $/ \mathrm{cm}^{2} \triangleq 0.1 \mathrm{n} \Omega \mathrm{cm}$.

The galvanomagnetic coefficient $P_{d}$ for defects introduced by cold working is determined as follows (4):

$$
P_{o}=P_{d}\left(Q_{d} / \rho_{o}\right)+P_{i}\left(\rho_{i} / \rho_{o}\right)-0.5\left|P_{d}-P_{i}\right| \rho_{d} \rho_{i} / \rho_{o}^{2}
$$

where $\rho_{0}=\rho_{d}+\rho_{i}$ and $P_{d}$ and $P_{i}$ the galvanomagnetic coefficients for dislocations and impurity atoms, respectively.

Fig. 1 illustrates the annealing behaviour of deformed Al wires. Annealing is complete at $620 \mathrm{~K}$. The annealing of $\oint_{0}$ exhibits one distinct revocery atage in the tempreature range of self-diffusion. Analytical checks on the reaction order of the annealing process of $\rho_{0}$ definitely exclude first order and second order kinetics. This points to the fact that the annealing between 300 and $620 \mathrm{~K}$ cannot be explained by a simple vacancy diffusion mechanism (5).

According to equation (1) we can evaluate the galvanomagnetic coefficient $\mathrm{P}_{\mathbf{i}}$ (in the low field region) due to impurity atoms, which yields 


$$
P_{i}=0.451(\mathrm{n} \Omega \mathrm{cm} / \mathrm{kG})^{2} \text { at } Q_{i}=1.932 \mathrm{n} \Omega \mathrm{cm} .
$$

From cold-worked samples which were annealed at room temperature we find in the low $\mathrm{H}$ region

$$
P_{0}=0.309(\mathrm{n} \Omega \mathrm{cm} / \mathrm{kG})^{2} \text { at } \oint_{0}=9.189 \mathrm{n} \Omega \mathrm{cm} \text {. }
$$

Utilizing equation (3) we find for the defects introduced by the cold work

$$
P_{d}=0.295(\mathrm{n} \Omega \mathrm{cm} / \mathrm{kG})^{2} \text { at } \varrho_{d}=7.257 \mathrm{n} \Omega \mathrm{cm} \text {. }
$$

Samples with different impurity content, $i . e$. with different $P_{i}$ values ranging from 0.4 to $0.55(\mathrm{n} \Omega \mathrm{cm} / \mathrm{kG})^{2}$ always yield

$$
\mathrm{P}_{\mathrm{d}}=0.29 \text { to } 0.30(\mathrm{n} \Omega \mathrm{cm} / \mathrm{kG})^{2} \text {. }
$$

Even at high magnetic fields the various defects (impurities, dislocations, vacancies) have a marked influence on the magnetoresistivity $\Delta \mathrm{g} / \mathrm{g}_{0}$ as already mentioned in (1). This is illustrated by the upper curve in Fig. 1. We observe two recovery steps, the first between 300 and $440 \mathrm{~K}$ the second between 540 and $620 \mathrm{~K}$.

For impurity atoms we find according to equation (2) a normalized high field coefficient:

$$
S=0.729 \text { at } \omega \tau \approx 10 .
$$

Defects introduced by cold work show the smaller $S=0.645$. Introducing, however, defects produced by quenching results in a higher $S$ between 0.68 and 0.95 . Here $S=0.68$ the pre-quench value of samples used in these experiments.

At present there is no theoretical calculation about the high field dependence of the magnetoresistivity on defects. It is likely that the investigation of magnetoresistivity annealing provides a simple and effective possibility to cover the region between point defect and large defect aggregates.

\section{Acknowledgement}

The author would like to thank Prof. R. Sizmann for his interest and helpful remarks.

\section{References}

(1) C. RAU, phys. stat. sol. (a) $\underline{13}, 207$ (1972).

(2) M. B. KASEN, Phil. Mag. 21, 599 (1970).

(3) F.R. FICKETT, Phys. Rev. B 3 , 1941 (1971).

(4) K. CLAUSECKER, phys. stat. sol. (b) $\underline{46}, 151$ (1971).

(5) K. RÖSCH, F. BELL, and R. SIZMANN, in : Report Jül. Conf, 2, Vol.1, 2, 1968 (p.444). 